\title{
As Diferentes Versões da Transformada de Helmert e suas Aplicações na Transformação entre Sistemas de Referência ${ }^{1}$
}

E.M. de SOUZA ${ }^{2}$, D.B.M. ALVES ${ }^{3}$, J.F.G. MONICO ${ }^{4}$, FCT, UNESP, 19060-900 Presidente Prudente, SP, Brasil.

\begin{abstract}
Resumo. Neste artigo objetiva-se descrever os conceitos fundamentais, dedução analítica e diferentes versões da transformada de Helmert. Essa transformada tem sido amplamente utilizada para realizar transformações entre sistemas de referência, pois permite que um conjunto de pontos em um sistema seja transformado para outro, utilizando translações, rotações e escalas. A utilização de sistemas de referência é muito importante para qualquer tipo de posicionamento. Entretanto, devido ao fato da crosta terrestre estar em constante movimento, as coordenadas das estações utilizadas na determinação do sistema de referência devem ser periodicamente recalculadas. Dessa forma, deve-se ter cautela na utilização e comparação de coordenadas das estações terrestres, consideradas conhecidas, para que estejam no mesmo referencial e também compatíveis em termos da época da realização do mesmo. Além disso, é apresentada uma aplicação de Geodinâmica em que as diferentes versões da Transformada de Helmert são comparadas.
\end{abstract}

\section{Introdução}

A transformada de Helmert tem sido amplamente utilizada para realizar transformações entre sistemas de referência. Essa transformada permite que um conjunto de pontos em um sistema seja transformado para outro, utilizando translações, rotações e escalas.

A utilização de sistemas de referência é muito importante para qualquer tipo de posicionamento. Entretanto, devido ao fato da crosta terrestre estar em constante movimento, as coordenadas das estações utilizadas na determinação do sistema de referência devem ser periodicamente recalculadas. Dessa forma, deve-se ter cautela na utilização e comparação de coordenadas das estações terrestres, consideradas conhecidas, para que estejam no mesmo sistema de referência e também compatíveis em termos da época da realização do mesmo.

O sistema de referência denominado ITRF (International Terrestrial Reference Frame) é utilizado internacionalmente. Mas como existem várias realizações desse sistema, é necessário utilizar um método para transformação entre os mesmos.

\footnotetext{
${ }^{1}$ Trabalho financiado pela FAPESP - Processos 03/12770-3 e 03/12771-0,

2 eniuce@yahoo.com.br, Programa de Pós Graduação em Ciências Cartográficas,

${ }^{3}$ danibarroca@yahoo.com.br, Programa de Pós Graduação em Ciências Cartográficas,

${ }^{4}$ galera@fct.unesp.br, Departamento de Cartografia.
} 
Logo, objetiva-se neste artigo descrever os conceitos fundamentais da transformada de Helmert para transformação entre sistemas de referência. Além disso, pretende-se mostrar as diferentes versões que essa transformada pode assumir, bem como sua dedução analítica e aplicações.

\section{ITRF}

Sempre na busca de definir sistemas de referência de alta precisão, a comunidade científica concebeu, na década de 80 , a primeira versão de um sistema de referência que seria a combinação das técnicas de posicionamento mais precisas e disponíveis naquele momento, sendo elas: VLBI (Very Long Baseline Interferometry), SLR (Satellite Laser Range) e LLR (Lunar Laser Range). Esse sistema de referência é designado por ITRFyy, onde o número yy especifica os dois últimos dígitos do último ano cujos dados contribuíram com a realização em consideração.

O ITRF é a materialização do ITRS (International Terrestrial Reference System) através do ajustamento de várias séries de coordenadas SSC (Set of Station Coordinates) e as respectivas matrizes variância-covariância. As SSC são disponibilizadas por diversos centros de análise que contribuem com o IERS (International Earth Rotation and Reference Systems Service) e estão espalhados por todo o globo. O resultado desse processo é uma lista de coordenadas e velocidades das estações que fazem parte de cada um dos ITRF yy, bem como os parâmetros de transformação entre as diversas SSC ([4] e [5]).

Uma estação ITRF é caracterizada pelas coordenadas $X, Y, Z$ (geocêntricas) com as respectivas velocidades, isto é, $V x, V y, V z$, numa determinada época $t$ de referência $t_{0}$. Utilizando a representação: $\vec{X}=[X, Y, Z]^{T}$ e $\vec{V}=[V x, V y, V z]^{T}$, a posição de um ponto sobre a superfície terrestre pode ser expressa na forma

$$
\vec{X}(t)=\vec{X}_{0}+\vec{V}_{0}\left(t-t_{0}\right)+\sum_{i} \Delta \vec{X}_{i},
$$

onde $\vec{X}_{0}$ e $\vec{V}_{0}$ são os vetores posição e velocidade na época de referência e $\Delta \vec{X}_{i}$ são as correções devido aos vários efeitos que alteram com o tempo, tais como o deslocamento de maré da Terra sólida, a carga dos oceanos e a carga da atmosfera [5].

Devido a contínua deformação da crosta terrestre, as coordenadas das estações são alteradas com o passar do tempo. Por isso, até hoje, já foram disponibilizadas diversas realizações do ITRS, embora este seja único por ser a preconização unívoca do sistema de referência. A primeira realização foi denominada ITRF0, em 1988. A solução do ITRF0 está publicada no IERS Technical Note 4 ([2] e [4]). Depois dessa realização inicial, outras realizações foram efetuadas, como o ITRF88, ITRF89, ITRF90, ITRF91, ITRF92, ITRF93, ITRF94, ITRF96, ITRF97, ITRF2000 até a mais recente denominada ITRF2005.

Para compatibilizar diferentes referenciais, considerando a evolução temporal das coordenadas, necessita-se de uma transformação que aplica simultaneamente as translações, rotações, fator de escala e respectivas taxas de variação com relação ao tempo, além da velocidade da estação. Ela é denominada transformada de Helmert 
generalizada [3]. A utilização da transformada de Helmert generalizada é necessária em aplicações de alta precisão. Caso contrário, a incerteza das coordenadas resultantes seria maior que as variações devido à inconsistência dos referenciais envolvidos. Conseqüentemente, as contribuições ao utilizar esse tipo de transformação seriam imperceptíveis [6].

\section{Trasnformada de Helmert}

A transformada de Helmert ([7] e [10]) pode ser descrita como sendo uma transformação de similaridade entre dois conjuntos quaisquer, e no caso deste artigo, tais conjuntos consistem nas coordenadas de diferentes realizações do sistema de referência ITRS.

\subsection{Transformada de Helmert generalizada}

Tomando as coordenadas de um ponto $P$ qualquer, associadas a um sistema de referência ITRF yy em uma época de referência $\left(t_{0}\right)$, pode-se obter suas coordenadas no sistema de referência ITRFzz em uma outra época $(t)$, com a aplicação da transformada de Helmert generalizada de 14 parâmetros, da seguinte forma ([6] e [9])

$$
\begin{gathered}
\vec{X}_{\text {ITRFzz }(t)}=\vec{T}+(1+s)(\varepsilon+I) \cdot\left(\vec{X}_{\text {ITRFyy }\left(t_{0}\right)} \vec{V}_{\text {ITRFyy }\left(t_{0}\right)}\left(t-t_{0}\right)\right)+ \\
\left.(\vec{T}+(1+s)(\dot{\varepsilon})+\dot{s}((\varepsilon)+(I))) \vec{X}_{\text {ITRFyy }\left(t_{0}\right)}\right) \cdot\left(t-t_{0}\right),
\end{gathered}
$$

onde

$$
\text { - } \varepsilon=\left[\begin{array}{ccc}
0 & \varepsilon_{z} & -\varepsilon_{y} \\
-\varepsilon_{z} & 0 & \varepsilon_{x} \\
\varepsilon_{y} & -\varepsilon_{x} & 0
\end{array}\right] \text { é uma matriz de rotação, sendo } \varepsilon_{x}, \varepsilon_{y} \text { e } \varepsilon_{z} \text { as }
$$

rotações diferenciais, em radianos, em torno dos eixos $X, Y$ e $Z$, respectivamente;

- $\vec{X}_{I T R F y y}$ e $\vec{X}_{I T R F z z}$ são as coordenadas $[X, Y, Z]^{T}$ de um ponto $P$ qualquer nos referenciais especificados;

- $\vec{V}_{I T R F y y\left(t_{0}\right)}=\left[V_{x}, V_{y}, V_{z}\right]^{T}$ é o vetor velocidade do ponto ITRFyy na época $t_{0}$ devido ao movimento da placa litosférica que o contém;

- $\vec{T}=\left[T_{x}, T_{y}, T_{z}\right]^{T}$ é o vetor com os parâmetros de translação do sistema de referência ITRF yy para o ITRF $z z$;

- $s$ é o fator de escala entre os dois referenciais;

- $\dot{\vec{T}}, \dot{\varepsilon}$ e $\dot{s}$ representam as variações em translação, rotação e fator diferencial de escala no ITRF yy com relação ao tempo;

- $\left(t-t_{0}\right)$ é o intervalo de tempo expresso em anos.

Vale ressaltar que as denotações ITRF yy e ITRF $z z$ representam dois sistemas de referência arbitrários, devendo ser substituidas na fórmula caso os sistemas de referência sejam outros.

Para obter uma melhor compreensão da equação (3.1), é mostrada a seguir a dedução analítica desa equação.

Como a Terra é um corpo não-rígido, as coordenadas de um ponto $P$ alteram-se com a taxa de variação, referenciada em ITRFyy, que é determinada a partir da 
variação média de sua posição com relação ao tempo. Assim, para um intervalo de tempo entre $t_{0}$ e $t$ tem-se

$$
\vec{V}_{I T R F y y\left(t_{0}\right)}=\frac{\vec{X}_{I T R F y y(t)}-\vec{X}_{I T R F y y\left(t_{0}\right)}}{\left(t-t_{0}\right)}
$$

$$
\vec{X}_{I T R F y y(t)}=\vec{X}_{I T R F y y\left(t_{0}\right)}+\vec{V}_{I T R F y y\left(t_{0}\right)}\left(t-t_{0}\right) .
$$

Da mesma forma, as coordenadas desse ponto no sistema de referência ITRF $z z$, em um instante $t$, são determinadas por

$$
\vec{X}_{I T R F z z(t)}=\vec{X}_{I T R F z z\left(t_{0}\right)}+\vec{V}_{I T R F z z\left(t_{0}\right)}\left(t-t_{0}\right) .
$$

De acordo com [1] a transformação entre sistemas de referência é dada por

$$
\vec{X}_{I T R F z z\left(t_{0}\right)}=\vec{T}+(1+s) R \vec{X}_{I T R F y y\left(t_{0}\right)},
$$

cuja derivada é

$$
\vec{V}_{I T R F z z\left(t_{0}\right)}=\dot{\vec{T}}+[(1+s) \dot{R}+\dot{s} R] \vec{X}_{I T R F y y\left(t_{0}\right)}+(1+s) R \vec{V}_{I T R F y y\left(t_{0}\right)} .
$$

Substituindo (3.5) e (3.6) na equação (3.4), tem-se

$$
\begin{gathered}
\vec{X}_{I T R F z z(t)}=\vec{T}+(1+s) R \vec{X}_{I T R F y y\left(t_{0}\right)}+ \\
{\left[\dot{\vec{T}}[(1+s) \dot{R}+\dot{s} R] \vec{X}_{\text {ITRFyy }\left(t_{0}\right)}+(1+s) R \vec{V}_{\text {ITRFyy }\left(t_{0}\right)}\right]\left(t-t_{0}\right) .}
\end{gathered}
$$

Associando os termos em comum, tem-se

$$
\begin{gathered}
\vec{X}_{I T R F z z(t)}=\vec{T}+(1+s) R\left[\vec{X}_{I T R F y y\left(t_{0}\right)}+\vec{V}_{\text {ITRFyy }\left(t_{0}\right)}\left(t-t_{0}\right)\right]+ \\
{\left[\dot{\vec{T}}+[(1+s) \dot{R}+\dot{s} R] \vec{X}_{\text {ITRFyy }\left(t_{0}\right)}\right]\left(t-t_{0}\right) .}
\end{gathered}
$$

Considerando que

$$
R=\varepsilon+I,
$$

e substituindo a equação (3.9) na equação (3.10), chega-se a equação (3.1) como se desejava.

$\mathrm{Na}$ equação da transformada de Helmert generalizada (equação (3.1)) pode-se notar a presença do termo

$$
(1+s)(\varepsilon+I)
$$

que pode ser expandido para

$$
\varepsilon+I+s \varepsilon+s I .
$$

Em muitas aplicações, a equação (3.11) pode ser simplificada por

$$
\varepsilon+(1+s) I=\left[\begin{array}{ccc}
(1+s) & \varepsilon_{z} & -\varepsilon_{y} \\
-\varepsilon_{z} & (1+s) & \varepsilon_{x} \\
\varepsilon_{y} & -\varepsilon_{x} & (1+s)
\end{array}\right]
$$


Isto ocorre, principalmente, devido ao fato do fator diferencial de escala $(s)$ ser constituído de um valor pequeno, de forma que a contribuição do produto do termo $s \varepsilon$ é insignificante para muitas aplicações [6].

Vale ressaltar que a transformada de Helmert generalizada é aplicada nos casos em que existem 7 parâmetros de transformação (3 translações, 3 rotações e 1 fator diferencial de escala) e mais 7 referentes as suas taxas de variação com relação ao tempo, totalizando 14 parâmetros, além das 3 componentes de velocidade $\left(V_{x}, V_{y}, V_{z}\right)$ da estação.

No entanto, existem casos em que as taxas de variação dos parâmetros não são disponíveis ou podem ser desprezadas por serem pequenas. Neste caso a transformada de Helmert generalizada (equação (3.1)) reduz-se a

$$
\vec{X}_{I T R F z z(t)}=\vec{T}+(1+s)(\varepsilon+1)\left(\vec{X}_{I T R F y y\left(t_{0}\right)}+\vec{V}_{I T R F y y\left(t_{0}\right)}\left(t-t_{0}\right)\right) .
$$

Dessa forma, nessa versão, aplica-se 3 translações, um fator diferencial de escala e 3 rotações, totalizando 7 parâmetros. Além desses, também faz parte desse tipo de transformação as componentes do vetor velocidade $\left(V_{x}, V_{y}, V_{z}\right)$.

\section{Fórmula Utilizada pelo IERS}

Uma outra possibilidade para realizar transformações entre sistemas de referência é utilizar a fórmula disponibilizada pelo IERS.

Para atualização dos parâmetros e coordenadas da estação da época inicial $t_{0}$ para uma certa época $t$, utiliza-se a seguinte equação

$$
P(t)=P\left(t_{0}\right)+\dot{P}\left(t-t_{0}\right),
$$

para um dado parâmetro ou coordenada $P$, cuja taxa de variação é $\dot{P}$.

Uma vez atualizados os parâmetros e coordenadas, deve-se realizar a transformação entre os sistemas de referência, utilizando a seguinte equação

$$
\vec{X}_{I T R F z z}=\vec{X}_{\text {ITRFyy }}+\vec{T}+\left[\begin{array}{ccc}
s & \varepsilon_{z} & -\varepsilon_{y} \\
-\varepsilon_{z} & s & \varepsilon_{x} \\
\varepsilon_{y} & -\varepsilon_{x} & s
\end{array}\right] \vec{X}_{\text {ITRFyy }}
$$

\section{Aplicações}

Nesta seção é apresentada uma aplicação onde é necessário realizar transformações entre diferentes referenciais. As transformadas de Helmert generalizada (14 parâmetros), simplificada (7 parâmetros) e a fórmula do IERS são aplicadas e os resultados comparados. Devido ao fato do ITRF2005 e o ITRF2000 serem os sistemas mais atuais, os parâmetros de transformação entre os mesmos (Tabela 1), disponíveis em http://itrf.ensg.ign.fr/ITRF, foram utilizados neste artigo. 
Tabela 1: Parâmetros de Transformação do ITRF 2005 para o ITRF 2000. As respectivas taxas de variação de $T$ são dadas em $\mathrm{mm} /$ ano e $s$ em 10 $-9 /$ ano.

\begin{tabular}{ccccccc}
\hline$T_{x}$ & $T_{y}$ & $T_{z}$ & $s$ & $\varepsilon_{x}$ & $\varepsilon_{y}$ & $\varepsilon_{z}$ \\
$m m$ & $m m$ & $m m$ & $10^{-9}$ & $0,001 ”$ & $0,001 ”$ & $0,001 ”$ \\
0,1 & $-0,8$ & $-5,8$ & 0,4 & 0,0 & 0,0 & 0,0 \\
\hline$\dot{T}_{x}$ & $\dot{T}_{y}$ & $\dot{T}_{z}$ & $\dot{s}$ & $\dot{\varepsilon_{x}}$ & $\dot{\varepsilon}_{y}$ & $\dot{\varepsilon}_{z}$ \\
$-0,2$ & 0,1 & $-1,8$ & 0,08 & 0,0 & 0,0 & 0,0 \\
\hline
\end{tabular}

\subsection{Referenciando uma estação pertencente ao ITRF 2005 no ITRF 2000}

Suponha que ao desenvolver uma atividade de Geodinâmica no ano de 2003 um pesquisador determinou a posição de uma estação com alta precisão. A técnica utilizada foi o GPS (Global Positioning System) e as coordenadas finais foram associadas ao sistema de referência ITRF 2000 (época 1997). Posteriormente essa coordenada foi determinada no ITRF 2005 (época 2000) utilizando a mesma técnica. Para que seja possível comparar os resultados de forma adequada, é necessário realizar uma transformação entre os referenciais.

Tabela 2: Coordenadas da estação BRAZ nos sistemas ITRF 2005 (obtida pelo usuário) e ITRF 2000 (disponível em http://itrf.ensg.ign.fr/ITRF_solutions /2000/sol.php).

\begin{tabular}{cccc}
\hline Sistema de ref. & $X$ & $Y$ & $Z$ \\
Epoca & $V_{x}$ & $V_{y}$ & $V_{z}$ \\
\hline ITRF2005 & 4115014,083 & $-4550641,541$ & $-1741444,022$ \\
2000 & 0,0002 & $-0,0046$ & 0,0124 \\
\hline ITRF2000 & 430711,408 & $-5099641,548$ & $-1699432,977$ \\
1997 & 0,0009 & $-0,0066$ & 0,0113 \\
\hline
\end{tabular}

Como essa situação é uma suposição, com o objetivo de ilustrar uma solução para o problema, foi utilizada a estação BRAZ (Brasília), cujas coordenadas são conhecidas nos dois referencias e respectivas épocas. A tabela 2 apresenta as coordenadas e velocidades da estação BRAZ.

Para referenciar as coordenadas do ITRF 2005 no ITRF 2000, serão aplicadas a transformada de Helmert generalizada, a simplificada e a fórmula do IERS. 


\subsubsection{Utilizando a transformada de Helmert generalizada (14 parâme- tros)}

Utilizando a equação (3.1), tem-se

$$
\begin{aligned}
& {\left[X_{\text {IT RF } 2000}\right]_{1997}=\left[\begin{array}{r}
0,0001 \\
-0,0008 \\
-0,0058
\end{array}\right]+\left(1+\left(0,4 \times 10^{-9}\right)\right) I_{3 \times 3}\left[\left[\begin{array}{r}
4115014,083 \\
-4550641,541 \\
-1741444,022
\end{array}\right]+\right.} \\
& \left.\left[\begin{array}{r}
0,0002 \\
-0,0046 \\
0,0124
\end{array}\right](1997-2000)\right]+\left[\begin{array}{r}
-0,0002 \\
0,0001 \\
-0,0018
\end{array}\right]+\left[\left(1+\left(0,4 \times 10^{-9}\right)\right) 0_{3 \times 3}+\right. \\
& \left.\left(0,08 \times 10^{-9}\right) I_{3 \times 3}\left[\left[\begin{array}{r}
4115014,083 \\
-4550641,541 \\
-1741444,022
\end{array}\right](1997-2000)\right]\right]=\left[\begin{array}{r}
4115014,083 \\
-4550641,529 \\
-1741444,059
\end{array}\right] \text {. }
\end{aligned}
$$

\subsubsection{Utilizando a transformada de Helmert (7 parâmetros)}

Utilizando a equação (3.13), tem-se

$$
\begin{gathered}
{\left[X_{\text {ITRF00 }}\right]_{1997}=\left[\begin{array}{r}
0,0001 \\
-0,0008 \\
-0,0058
\end{array}\right]+\left(1+\left(0,4 \times 10^{-9}\right)\right) I_{3 \times 3}} \\
{\left[\left[\begin{array}{r}
4115014,083 \\
-4550641,541 \\
-1741444,022
\end{array}\right]+\left[\begin{array}{r}
0,0002 \\
-0,0046 \\
0,0124
\end{array}\right](1997-2000)\right]=\left[\begin{array}{r}
4115014,084 \\
-4550641,529 \\
-1741444,065
\end{array}\right] .}
\end{gathered}
$$

\subsubsection{Utilizando a fórmula do IERS}

Para utilizar a fórmula do IERS, primeiramente é necessário atualizar os parâmetros (translações, rotações e escala) e as coordenadas obtidas na época 2000 para a época 1997 (equação (4.1)). Assim, atualizando as coordenadas de acordo com as velocidades da estação, tem-se

$$
\begin{aligned}
{\left[\begin{array}{c}
X_{I T R F 05} \\
Y_{I T R F 05} \\
Z_{I T R F 05}
\end{array}\right]_{1997} } & =\left[\begin{array}{r}
4115014,083 \\
-4550641,541 \\
-1741444,022
\end{array}\right]+\left[\begin{array}{r}
0,0002 \\
-0,0046 \\
0,0124
\end{array}\right](1997-2000) \\
& =\left[\begin{array}{r}
4115014,082 \\
-4550641,527 \\
-1741444,059
\end{array}\right] .
\end{aligned}
$$

Atualizando os parâmetros de translação e escala

$$
\left[\begin{array}{l}
T_{x} \\
T_{y} \\
T_{z}
\end{array}\right]=\left[\begin{array}{r}
0,0001 \\
-0,0008 \\
-0,0058
\end{array}\right]+\left[\begin{array}{r}
-0,0002 \\
0,0001 \\
-0,0018
\end{array}\right](1997-2000)=\left[\begin{array}{r}
0,0007 \\
-0,0011 \\
-0,0004
\end{array}\right],
$$

e, no caso da escala,

$$
[s]_{1997}=0,4 \times 10^{-9}+0,08 \times 10^{-9}(1997-2000)=0,16 \times 10^{-9} .
$$


Como as rotações e as respectivas variações são nulas, não é necessário atualizálas.

Em seguida, é necessário realizar a transformação entre os referenciais (equação $(4.2))$

$$
\begin{gathered}
{\left[\begin{array}{c}
X_{\text {ITRF } 00} \\
Y_{\text {ITRF } 00} \\
Z_{\text {ITRF } 00}
\end{array}\right]_{1997}=\left[\begin{array}{r}
4115014,082 \\
-4550641,527 \\
-1741444,059
\end{array}\right]+\left[\begin{array}{r}
0,0007 \\
-0,0011 \\
-0,0004
\end{array}\right]+} \\
0,16 \times 10^{-9} I_{3 \times 3}\left[\begin{array}{r}
4115014,082 \\
-4550641,527 \\
-1741444,059
\end{array}\right]=\left[\begin{array}{c}
4115014,083 \\
-4550641,529 \\
-1741444,059
\end{array}\right] .
\end{gathered}
$$

\subsubsection{Comparando com as coordenadas conhecidas}

A discrepância entre as coordenadas calculadas (seções 4.1.1, 4.1.2 e 4.1.3) e as conhecidas (Tabela 2) da estação BRAZ na época 1997 são apresentadas na Figura 1a. Já as resultantes dessas discrepâncias são ilustradas na Figura 1b.

Observando as Figuras 1a e 1b pode-se verificar que as discrepâncias apresentadas não chegam a $0,6 \mathrm{~cm}$. Além disso, percebe-se que os resultados apresentados pela transformada de Helmert generalizada são iguais aos obtidos pela fórmula do IERS.

É importante salientar que os resultados obtidos pela fórmula de Helmert de 7 parâmetros foram um pouco piores $(0,14 \mathrm{~cm}$ na resultante). Isso se deve à simplificação da fórmula (equação (3.13)). Portanto, percebe-se que dependendo da aplicação a utilização das taxas de variação é dispensável.

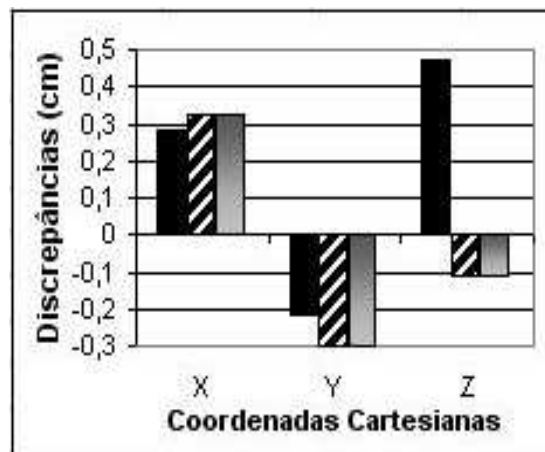

(a)

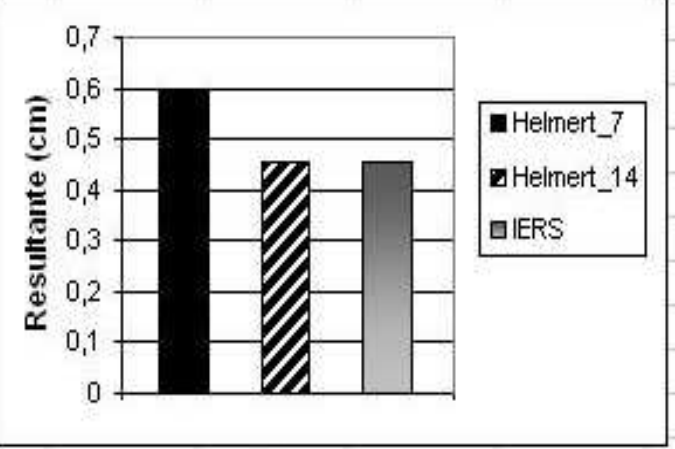

(b)

Figura 1: Discrepâncias (a) e Resultante (b) das coordenadas conhecidas com as obtidas pela transformada de Helmert ( 7 e 14 parâmetros) e fórmula do IERS. 


\title{
6. Conclusão
}

Neste artigo foram apresentados os detalhes da transformada de Helmert, envolvendo sua dedução analítica e as diferentes versões que ela pode assumir.

Tal transformada deve ser aplicada para os casos em que são empregados métodos de posicionamento que proporcionem alta precisão e estejam associados a re- ferenciais bem definidos e materializados, como as várias realizações do ITRS, embora este seja único por ser a preconização unívoca do sistema de referência.

Os parâmetros utilizados ao aplicar a transformada de Helmert generalizada em trabalhos que se utiliza o posicionamento por satélites (como o GPS), apesar de ser de pequena dimensão, são importantes para preservar a qualidade dos resultados. Isto ocorre devido a melhoria considerável da acurácia dos resultados obtidos no posicionamento por satélites, que evoluíram nas últimas décadas e se tornaram sensíveis a pequenas variações. Anteriormente, tais parâmetros só tinham significado para resultados obtidos por técnicas mais avançadas, como o VLBI e o SLR, muito utilizadas em geodinâmica.

Foi apresentada uma aplicação envolvendo situações que necessitam de transformações entre referenciais. As discrepâncias entre as coordenadas em dois referenciais diferentes foram calculadas. Tal procedimento é importante, pois, fornece subsídios para prever a dimensão dos erros que podem ser cometidos nos trabalhos que os envolvem.

\begin{abstract}
The aim of this paper is to describe the fundamental concepts, analytical deduction and different versions of the Helmert Transform. This transform has been widely used to accomplish transformations between reference systems, because it allows transforming a point set in a system to another one using translations, rotations and scales. The use of reference systems is very important for any kind of positioning. However, due to the terrestrial crust be in constant movement, the station coordinates used in the reference system determination should be recomputed periodically. Thus, one should have caution in the use and comparison of terrestrial station coordinates, considered known, for that they be in the same referential and realization epoch. Furthermore, a Geodynamic application is presented where the Helmert Transform and its different versions are compared.
\end{abstract}

\section{Referências}

[1] Y. Bock, Reference System, in "GPS for Geodesy" (A. Kleusber e P.J.G. Teunissen, eds), pp. 1-41, Berlin, Verlag, 1998.

[2] C. Boucher, Z. Altamini, "Evolution of the Realization of the Terrestrial Reference System - IERS Technical Note 4", IERS/CB, Paris, 1990.

[3] T. Gregorius, "How it Works...GIPSY OASIS II", Departament of Geomaties University of Newcastle upon Tyne, 1996.

[4] D.D. McCarthy, G. Petit, "IERS Conventions: IERS Technical Note 32". Frankfurt am Main: Verlag des Bundesamts für Kartographie und Geodäsie, 2004. 
[5] J.F.G. Monico, "Posicionamento pelo NAVSTAR - GPS: Descrição, fundamentos e aplicações". São Paulo, UNESP, 2000.

[6] L.F. Sapucci, J.F.G. Monico, Transformação de Helmert Generalizada no posicionamento de alta precisão: fundamentação teórica e aplicações. Revista Brasileira de Geofísica, 18 (2000).

[7] J. Sevcik, Weak nonlinearity in a model which arises from the helmert transformation. Applications of Mathematics, 48 (2003), 161-174.

[8] T. Soler, A compendium of transfomation formulas useful in GPS work. National Geodetic Survey, Journal of Geodesy, 72 (1998), 482-490.

[9] T. Soler, Transformações Rigorosas entre sistemas de Referência de coordenadas: Aplicação ao GPS (ITRF, WGS84) e GLONASS (PZ90). GeoConvergência, (1999), 30-38.

[10] G.A. Watson, Computing Helmert transformations. Journal of Computational and Applied Mathematics, 197 (2006), 387-394. 